\title{
FUNGAL PROFILE OF SOME SELECTED EGYPTIAN MEDICINAL PLANTS AND SPICES AND ESTIMATION OF MYCOTOXIN PRODUCTION
}

(Received: 12.3.2012)

\author{
By \\ F.H.Eltahan, Y. M. Shetaia* and M. F. Abd Elaziz** \\ Agriculture Research Center,Giza., and *Microbiology Department,Faculty of Science,Ain Shams \\ University, Egypt **Centeal laboratory of residue analysis of pesticides and heavy metals in food. \\ Agriculture Research Center, Giza
}

\begin{abstract}
Hundred and fifty samples of five medicinal plants and spices ( Basil, Chamomile,Dry mint,Marjoram and Fennel) were screened for the isolation of mold and yeast flora. The prevalence of molds ranged from $10.04 \%$ in Fennel to $33.95 \%$ in Dry mint. Aspergillus niger was the most prevalent mold. It was represented by 428 isolates (32.08\%) in Basil, 190 isolates (28.83\%) in Chamomil, 729 isolates (34.45\%) in Dry mint, 546 isolates (36.45\%)in Marjoram and 45 isolates (7.19\%) in Fennel. The prevalence of yeasts ranged from $0.0 \%$ in Basil to $38.9 \%$ in Chamomile. Saccharomyces cerevisiae was the most prevalent yeast. The anti fungal activity of ethanolic extracts of Cinnamon, Curcuma and Ginger were estimated against the derived molds and yeasts isolated from the selected spices and medicinal plants( 60 isolates of molds and 18 isolates of yeast). All mold isolates were resistant to the Curcuma and Ginger extracts. In case of Cinnamon extract, only nine mold isolates were intermediate, while eighteen mold isolates were sensitive. All yeast isolates were resistant to the ethanolic extract of each of Curcuma and Cinnamon, while the majority of yeast isolats were sensitive to Ginger extract. Seven mold isolates out of sixty produced mycotoxins(11.7\%). Aspergillus flavus from Marjoram produced aflatoxin B1 $(160 \mu \mathrm{g} / \mathrm{ml})$ and aflatoxin B2 $(50 \mu \mathrm{g} / \mathrm{ml})$, while A.flavus from Basil produced aflatoxin G1 $(25 \mu \mathrm{g} / \mathrm{ml})$. Each of A.glaucus, A.versicolor from Dry mint and A.nidulans from Basil produced zeralenone.
\end{abstract}

Key words: antifungal activity, fungal flora, medicinal plants, mycotoxins.

\section{INTRODUCTION}

Medicinal plants and herbs were known to modern and ancient civilization, particularly ancient Egyptians, for their healing properties(ElSawi, 2000).Medicinal plants are of increasing importance in Egypt, they were used as human remedies and raw materials for pharmaceutical and cosmetic industries. The source of contamination of medicinal plants referred to the exposure of public as consumers during cultivation and the unhygienic ways of storage. Bacterial or fungal flora play an important role in the contamination of medicinal plants. These microbial flora were responsible for lowering yield of medicinal plants(El Tahan \& Fahmy, 2005). Fungi are the predominant contaminants of spices (Kneifel \& Berger, 1994), but most of such microbial populations were probably regarded as commensal residents on the plants that survived drying and storage. This referring to the uncontrolling of humidity during storage (Aziz et al., 1998). Strains and species of Aspergillus flavus group were widely distributed in many different therapeutic herbal medicines. Aspergillus sp., Penicillium sp., Rhizopus sp. and Mucor sp. are regular elements of spice fungal flora, while yeast isolates were absent (Wojcik and Jakubowska, 2005). Walker, (2002) stated that prevention of contamination at source is considered to be the most effective public health measure. Secondary metabolites of certain ubiquitous moulds growing on dried herbs may lead to toxic effects(El Tahan and Taha,1996). The chlorinated isocoumarin compound, ochratoxin A (OTA), together with some related derivatives (ochratoxins B, C, alpha, beta) were produced by Penicillium verrucosum and by several species of Aspergillus, most notably Aspergillus ochraceus. Penicillium verrucosum was the principal source of OTA contamination of 
stored foods in temperate climates, while Aspergillus sp. predominated in warmer countries(Walker, 2002). The contamination of raw materials of spices and medicinal plants by microorganisms is one of the major reasons for decline any country's share in the global spices and medicinal plants market. Also,the risk assessment of the microbial load of medicinal plants has become an important subject in the establishment of modern Hazard Analysis and Critical Control Point (HACCP) schemes( Kneifel et al., 2002). Accordingly, the aim of this study was to determine the fungal profile(mold,yeast) in 150 samples of the selected five spices and medicinal plants( Basil, Marjoram, Drymint, Fennel, Chamomile) and to evaluate the capability of sixty molds to produce mycotoxins. Also, this study was concerned with the estimation of the antifungal activity of ethanolic extract of some spices and medicinal plants( curcuma, cinnamon and ginger)against fungal flora (mold,yeast) isolated from the selected medicinal plants.

\section{MATERIALS AND METHODS}

\subsection{Spices and medicinal plant samples}

Thirty samples of each of the selected five spices and medicinal plants ( Basil, Chamomile, Dry mint, Marjoram and Fennel) were brought to the Central Laboratory for food and feed [Agriculture Research Center] for making chemical and microbial analysis before they were exported to European countries. These samples (150 samples) were screened for the isolation of fungal flora(molds \& yeasts).

\subsection{Preparation of medicinal plant samples}

Ten grams of each of the spices and medicinal plant samples were mixed with $90 \mathrm{ml}$ diluent (maximal recovery diluent) in the mixer (Stomacher 400). The mixing process was extended for $10 \mathrm{~min}$. Ten fold serial dilutions were prepared. Six levels spacing one logarithmic unit were investigated by pipetting one $\mathrm{ml}$ from each level into duplicate sterile petri dishes containing the specific medium.

\subsection{Isolation of molds and yeasts (ISO 21527 2001)}

Each sample of the medicinal plants $(1 \mathrm{ml})$ was mixed carefully with $15 \mathrm{ml}$ of Dichlorane $18 \%$ Glycerol agar medium (DG18 ISO /CD 21527(2001) and allowed to solidify. The plates were incubated at $30+2{ }^{\circ} \mathrm{C}$ for $48 \mathrm{hr}$ for the isolation of yeast and for 3- 4 days for the isolation of molds. After three days of incubation, the colonies in each plate were counted. The plates containing fewer than 150 colonies were discarded. A control plate with $15 \mathrm{ml}$ medium was prepared to check the sterility. The identification of the fungal isolates was carried out on the basis of growth and microscopic morphology using the following universal manuals (Samson, 1974; John, 1979 and Kendrick, 2000), while the yeast isolates were identified using the routine laboratory methods recommended by (Wickerham, 1951; Lodder, 1971; Ahearn, 1978 and Barnett et al., 2000).

\subsection{Statistical analyses}

All data were subjected to statistical analysis of variance (F-test) "one way ANOV". It was noted that if the means of the subgroups are greatly different, the variance of the combined groups is much larger than the variance of the separate groups. The analysis of variance format for the differences in means is based on this fact.Duncan's multiple range test is one of the multiple- comparison procedures. It uses the " $\mathrm{t}$ " distribution corresponding to the number of degrees of freedom for error mean square.The significance of the measured data was considered as follows :

not significant (N.S) when $\mathrm{P}>0.05$

significant $(\mathrm{S})$ when $\mathrm{P}<0.05$

highly significant(H.S) when $\mathrm{P}<0.01$

where $\mathrm{P}$ is the probability (reflect of null hypothesis). Details of the formulae used are given by (Armitage, 1971).

2.5. Ethanolic extraction of the medicinal plants (cinnamon, curcuma and ginger) and estimation of its antifungal activity(Velickvic et al., 2002)

Fifty grams of the medicinal plants were added to $500 \mathrm{ml}$ of ethanol $(70 \%)$ and left for 2 days. The extract was filtered using Buchner filter. The filtrate was added into Rota vapor to evaporate alcohol and then left in the incubator at $44.5^{\circ} \mathrm{C}$ to evaporate the remaining water. The antifungal activity was estimated using the hole plate diffusion technique (Clark et al., 1981). Muller Hinton agar medium (g/L);Beef infusion(300), Casein hydrolysate(17.5), starch(1.5), Agar(17.0), $\mathrm{pH} / 7.3 \pm 0.2$ was prepared and allowed to solidify in plates. From the fungal flora of the selected five spices and medicinal plants, the suspensions of eighteen Yeast isolates and sixty mold isolates were prepared and matched with a McFarland 0.5. The suspensions were swabbed on the surface of Hinton agar medium using sterile cotton swab. Each of the ethanolic extract of the medicinal plants (Cinnamon,Curcuma and Ginger) was added to each hole plate and allowed to diffuse at room 
temperature for 20 minutes. The plates were incubated aerobically overnight at $30^{\circ} \mathrm{C}$. The antifungal activity was recorded as the diameter of the resulting inhibition zones measured in millimeters. According to Bauer et al., (1966), the microbicidal activity was classified into resistant if the diameter of inhibition zone is less than $8 \mathrm{~mm}$. Intermediate $(9-11 \mathrm{~mm})$ and sensitive $(\geq 12$ $\mathrm{mm})$.

\subsection{Mycotoxins production}

Sixty mould isolates from the selected five medicinal plants (Majoram, Dry mint, Chamomile, Basil and Fennel) were evaluated for the production of Aflatoxines, Zerianone and Ochratoxines. The inoculum was prepared by inoculating potato dextrose agar medium (PDA) with the selected mould isolates and incubated for 7 to 21 days at $28^{\circ} \mathrm{C}$. Flasks of liquid yeast $\operatorname{media}(\mathrm{g} / \mathrm{l})$; yeast extract(20), sucrose(200), magnesium sulphate $(0.2)$,calcium carbonate (0.2), $\mathrm{pH} 7$. were inoculated with the selected mould isolates and incubated at $15^{\circ} \mathrm{C}$ in the first day, at $21^{\circ} \mathrm{C}$ in the $2^{\text {nd }}$ day and at $28^{\circ} \mathrm{C}$ in the $3^{\text {rd }}$ day till the end of incubation period (from 15 days to 3 weeks). The flasks were steamed by autoclaving at $121^{\circ} \mathrm{C}$ for $15 \mathrm{~min}$ to destroy the fungus and then filtered to separate the spores from the liquid media (El Tahan and Taha, 1996).

\subsection{Qualitative assay of mycotoxins}

Ten $\mathrm{ml}$ of the filterate were shaked in separating funnel with $250 \mathrm{ml}$ chloroform for 30 $\mathrm{min}$. The chloroform layer was filtered using filter paper (Whatman no. 1), anhydrous sodium sulfate (25g) was added to remove water. The filterate was clarified using buchner funnel and concentrated under vacuum using rotary evaporator. The residue was made up to $10 \mathrm{ml}$ with chloroform for chromatography on thin-layer plates. 1,2,3,4,5 $\mu \mathrm{l}$ of the standers of aflatoxin (B1,B2, G1, G2 and zeralenone) were spotted on thin layer plates and developed on ether-methanolwater(96-3-1). The spots were examined under U.V. lamb at wave length of $365 \mathrm{~nm}(\mathrm{El}$ Tahan and Taha,1996). The quantitative performance of aflatoxin B1, B2 and G1 was carried out in the central laboratory for food and feed [Agriculture Research Center] using TLC Denistometer schmatzo 9000.

\section{RESULTS AND DISCUSSION}

\subsection{Prevalence and frequency of the isolated} molds and yeasts from themedicinal plants

The results in Table (1) show the prevalence of molds in different herbs, which ranged from $10.04 \%$ in Fennel to $33.95 \%$ in Dry mint, while the highest prevalence of yeasts (38.9\%) was represented in Chamomile (Table, 3). These results coincide with Martins et al., (2001)who revealed that many yeasts were found in Chamomile and Dry mint. From the total samples (150), twelve fungal isolates were detected; seven species of Aspergillus [A. glaucus (13.48\%), A.versicolor (3.05\%), A.nidulaus (7.78\%),A.niger (31.09\%), A.flavus (3.29\%), A.terreus (1.97\%) and A.flavipes (2.04\%)], Alternaria alternata (7.24\%), Acremonium strictum(23.34\%), Rhizopus orrhizus (2.15\%), Fusarium solni(4.09\%) and Absidia corymbifeza (0.48\%) (Table,1). Aspergillus niger was the most prevalent mold. It was represented by four hundred and twenty eight isolates (32.08\%) in Basil, 190 isolates (28.83\%) in Chamomile, 729 isolates (34.45\%) in Dry mint, 546 isolates $(36.45 \%)$ in Marjoram and 45 isolates (7.19\%) in Fennel. Mandeel (2005) found that Penicillium sp was the highly prevalent mold in Chamomile, Dry mint samples. Hashem and Alamri, (2010) found that the ginger samples were the most heavily contaminated spice samples and Aspergillus, Penicillium, and Rhizopus were the most predominant fungal isolates. Eighteen yeast isolates were isolated from the collected 30 samples of each of the selected five spices and medicinal plants (Marjoram, Dry mint, Chamomile, Basil and Fennel). Seven yeast species were detected ; Saccharomyces rosinii (1.3\%), Candida rugosa (2.0\%), Debaromyces hansenii (2.0\%), Lipomyces libofore (1.3\%), Trichosporon jirovecii (0.7\%), Saccharomyces cerevisiae $(2.7 \%)$ and Candida glabrata $(2.0 \%)$ (Table 3). Saccharomyces cerevisiae was the most prevalent yeast. It was represented by one isolate (3.3\%) in Chamomile, three isolates in Fennel (10.0\%). In the Basil samples, 1000, 300 and 34 mold isolates were isolated.Ten mold species were detected; Aspergillus glaucus (22.86\%), A.versicolor (4.27\%), A.nidulaus (15.67\%), A. niger (32.08\%), A. flavus (4.05\%), A. terreus (16.34\%), Alternaria alternata (0.60\%), Acremonium strictum (1.65\%), Rhizopus orrhizus $(1.35 \%)$ and Fusarium solani (1.12\%) (Table, 1). No yeast isolates were detected in Basil samples (Table, 3). The highest number of mould isolates was represented in Dry mint. Eleven mold species were identified as; Aspergillus glaucus $(7.75 \%)$, Aspergillus versicolor (4.82\%), Aspergillus nidulaus (1.89\%) , Aspergillus niger $(34.45 \%)$, Aspergillus flavus(3.64\%), Aspergillus terreus (4.82\%) Aspergillus flavipes(5.43\%), Alternaria alternate( $7.94 \%)$, Acremonium strictum(20.42\%), Rhizopus orrhizus(1.04\%) and Fusarium solani 
$(7.80 \%)($ Table, 1$)$. One yeast isolate was identified as Candida rugosa (3.3\%) (Table, 3). Martins et al., (2001) and Abou-Donia, (2008) reported that, Fusarium sp., Penicillium sp., Aspergillus flavus and $A$. niger were the predominant fungal isolates in almost all medicinal plant samples and many yeasts were found in Chamomile and Dry mint. In Marjoram samples, 1000, 498 mold isolates were isolated ; Twelve mold species were detected; Aspergillus glaucus $(19.69 \%), \quad A . \quad$ versicolor $(1.27 \%), A$. nidulaus $(7.74 \%), \quad A . \quad$ niger $(36.45 \%)$, A.flavus(2.40\%), A.terreus $(0.20 \%)$ A. flavipes (0.53\%), Alternaria alternate( $3.14 \%)$, Acremonium strictum (22.83\%), Rhizopus orrhizus $(0.87 \%)$, Fusarium solani $(2.94 \%)$ and Absidia corymbifeza (1.94\%) (Table, 1). Three yeast isolates were identified as Saccharomyces rosinii $(3.3 \%)$, Lipomyces libofore $(3.3 \%)$, and Candida glabrata (10.0\%) (Table 3). In Chamomile,659 mold isolates were isolated. Twelve mold species were identified as; Aspergillus glaucus(7.28\%), A.versicolor $(1.21 \%)$, A.nidulaus $(15.78 \%)$,

A.niger $(28.83 \%)$, A.flavus $(1.67 \%), \quad$ A.terreus $(1.37 \%) \quad$ and A.flavipes $(0.30 \%)$, Alternaria alternate $(13.05 \%)$, Acremonium strictum (23.98\%), Rhizopus orrhizus(1.67\%), Fusarium solni(4.70\%) and Absidia corymbifeza $(0.15 \%)$ (Table, 1$)$. The highest yeast count was detected in Chamomile; five yeast species were identified (23.3\%); Candida rugosa(6.6\%), Debaromyces hansenii(6.6\%), Lipomyces libofore(3.3\%), Trichosporon jirovecii( $3.3 \%$ ) and Saccharomyces cerevisiae (3.3\%) (Table 3). In Fennel, the lowest total fungal count was detected, ten mold species were identified; A.glaucus (4.47\%), A.versicolor (0.65\%), A.nidulaus (2.56\%) A.niger (7.19\%), A.flavus $(4.31 \%)$, A.terreus (0.16\%), A.flavipes (0.32\%), Alternaria alternate (20.45\%), Acremonium strictum (48.72\%) and Rhizopus orrhizus (11.18\%) (Table 1). Three yeast species were identified as Saccharomyces rosinii( $3.3 \%)$, Debaromyces hansenii(3.3\%)and Saccharomyces cerevisiae $(10.0 \%)$ (Table 3 ). The high levels of microbial contamination in spices and herbs were reported by many studies and suggested a need for better control in all aspects of the production, processing and usage of these products to prevent potential food spoilage and food-borne illnesses(McKee, 1995). Quality control of the exported and imported food products is a vital process. Herb-exporting countries will receive more revenues following the widening market for herbal products in developed countries(Dubey et al., 2008). The high prevalence of fungal contamination in herbs and spices was documented and referred to the unscientific methods of collection, storage, transportations and congenial climatic condition(Dubey et al., 2008). The results in Table (2) show the significant difference which was represented by the difference in letters (Duncan grouping). A highly significant $\mathrm{p}$ values were obtained with Aspergillus glaucus, A.versicolor,A.flavus (.001,.003 and .001, respectively). According to Duncan test ,in each row any two means taking the same letter there is no significant difference between them).

\subsection{Estimation of antifungal activity of the} ethanolic extracts of three medicinal plants (curcuma, cinnamon and ginger)

In this study,the ethanolic extracts of three medicinal plants (Curcuma, Cinnamon and Ginger) were used to inhibit the growth of the fungal contaminants in the selected five spices and medicinal plants. All mould isolates were resistant to the Curcuma and Ginger extracts( the inhibition zones ranged from 1-3mm)(Table 4). In the case of Cinnamon extract, nine mold isolates were intermediate ( the inhibition zones ranged from $9-11 \mathrm{~mm}$ ), eighteen mold isolates were sensitive (the inhibition ranged from $12-22 \mathrm{~mm}$ ) and other mold isolates were resistant to Cinnamon extract (Table, 3). Simić et al. (2004) reported that the trans-cinnamaldehyde is the essential oil of Cinnamomum zeylanicum, which showed the strongest antifungal activity. While the GC and GC-MS chemical analysis of volatile oils of Curcuma longa, C. zedoaria, C. aromatica and C. amada, revealed the presence of ar-turmerone, ar-turmerol, myrcene, $\beta$ - pinene, cineol, cymene, $\alpha$-phellandrene. These compounds are used as antifungal materials (Singh et al., 2002). All yeast isolates were resistant to the ethanolic extract of each of Curcuma and Cinnamon (the inhibition zone ranged from one to six $\mathrm{mm}$ ). In the case of ethanolic extract of Ginger, only two yeast isolates were intermediate (Lipomyces lipofore and Debaromyces hansenii) with inhibition zone of $10 \mathrm{~mm}$. Other yeast isolates were sensitive with inhibition zones ranged from $12 \mathrm{~mm}$ to $30 \mathrm{~mm}$ (Table, 4). These results matched with Lopez et al. (2005)who tested the antimicrobial activity of essential oils of cinnamon and ginger against Candida albicans and two moulds( Penicillium islandicum and Aspergillus flavus)and found that Cinnamon gave the strongest inhibition in the case of molds, while ginger gave the strongest inhibition in the case of yeasts. It is clear that 
Table (1): Frequency and percentage of the isolated molds from the collected thirty samples of each of the selected five spices and medicinal plants (basil, chamomile, dry mint, marjoram and fennel).

\begin{tabular}{|c|c|c|c|c|c|c|c|c|c|c|c|c|}
\hline \multirow{2}{*}{$\begin{array}{l}\text { Type of commodity } \\
\text { Mold isolates }\end{array}$} & \multicolumn{2}{|c|}{$\begin{array}{c}\text { Basil } \\
\text { (30 samples) } \\
\end{array}$} & \multicolumn{2}{|c|}{$\begin{array}{l}\text { Chamomile } \\
\text { (30 samples) }\end{array}$} & \multicolumn{2}{|c|}{$\begin{array}{c}\text { Dry mint } \\
\text { (30 samples) }\end{array}$} & \multicolumn{2}{|c|}{$\begin{array}{c}\text { Marjoram } \\
\text { (30 samples) }\end{array}$} & \multicolumn{2}{|c|}{$\begin{array}{c}\text { Fennel } \\
\text { (30 samples) }\end{array}$} & \multicolumn{2}{|c|}{$\begin{array}{c}\text { Total } \\
\text { (150 samples) }\end{array}$} \\
\hline & $\begin{array}{l}\text { No. of } \\
\text { isolates }\end{array}$ & $\%$ & $\begin{array}{c}\text { No. of } \\
\text { isolates }\end{array}$ & $\%$ & $\begin{array}{l}\text { No. of } \\
\text { isolates }\end{array}$ & $\%$ & $\begin{array}{l}\text { No. of } \\
\text { isolates }\end{array}$ & $\%$ & $\begin{array}{l}\text { No. of } \\
\text { isolates }\end{array}$ & $\%$ & $\begin{array}{l}\text { No. of } \\
\text { isolates }\end{array}$ & $\%$ \\
\hline Aspergillus glaucus & 305 & 22.86 & 48 & 7.28 & 164 & 7.75 & 295 & 19.69 & 28 & 4.47 & 840 & 13.48 \\
\hline Aspergillus versicolor & 57 & 4.27 & 8 & 1.21 & 102 & 4.82 & 19 & 1.27 & 4 & 0.64 & 190 & 3.05 \\
\hline Aspergillus nidulaus & 209 & 15.67 & 104 & 15.78 & 40 & 1.89 & 116 & 7.74 & 16 & 2.56 & 485 & 7.78 \\
\hline Aspergillus niger & 428 & 32.08 & 190 & 28.83 & 729 & 34.45 & 546 & 36.45 & 45 & 7.19 & 1938 & 31.09 \\
\hline Aspergillus flavus & 54 & 4.05 & 11 & 1.67 & 77 & 3.64 & 36 & 2.40 & 27 & 4.31 & 205 & 3.29 \\
\hline Aspergillus flavipes & 0 & 0.00 & 2 & 0.30 & 115 & 5.43 & 8 & 0.53 & 2 & 0.32 & 127 & 2.04 \\
\hline Alternaria alternate & 22 & 1.65 & 86 & 13.05 & 168 & 7.94 & 47 & 3.14 & 128 & 20.45 & 451 & 7.24 \\
\hline Acremonium strictum & 218 & 16.34 & 158 & 23.98 & 432 & 20.42 & 342 & 22.83 & 305 & 48.72 & 1455 & 23.34 \\
\hline Rhizopus orrhizus & 18 & 1.35 & 11 & 1.67 & 22 & 1.04 & 13 & 0.87 & 70 & 11.18 & 134 & 2.15 \\
\hline Fusarium solani & 15 & 1.12 & 31 & 4.70 & 165 & 7.80 & 44 & 2.94 & 0 & 0.00 & 255 & 4.09 \\
\hline Absidia corymbifeza & 0 & 0.00 & 1 & 0.15 & 0 & 0.00 & 29 & 1.94 & 0 & 0.00 & 30 & 0.48 \\
\hline Total & 1334 & 21.40 & 659 & 10.57 & 2116 & 33.95 & 1498 & 24.03 & 626 & 10.04 & 6233 & \\
\hline
\end{tabular}

Table (2): Mean value of isolated molds from the collected thirty samples of each of the selected five spices and medicinal plants (basil, chamomile, dry mint, marjoram and fennel).

\begin{tabular}{|c|c|c|c|c|c|c|c|}
\hline \multirow{2}{*}{$\begin{array}{l}\text { Type of commodity } \\
\text { Mold isolates }\end{array}$} & \multirow[t]{2}{*}{ Basil } & \multirow[t]{2}{*}{ Chamomile } & \multirow[t]{2}{*}{ Dry mint } & \multirow[t]{2}{*}{ Marjoram } & \multirow[t]{2}{*}{ Fennel } & \multicolumn{2}{|c|}{ F-test } \\
\hline & & & & & & F-value & P-value \\
\hline 1-Aspergillus glaucus & 101.66 a & $16.00 \mathrm{c}$ & $59.33 \mathrm{~b}$ & 98.33 a b & $9.33 \mathrm{c}$ & 12.071 & 0.001 \\
\hline 2-Aspergillus versicolor & $19.00 \mathrm{~b}$ & $2.66 \mathrm{c}$ & $35.33 \mathrm{a}$ & $6.33 \mathrm{bc}$ & $1.33 \mathrm{c}$ & 8.850 & 0.003 \\
\hline 3-Aspergillus nidulaus & $69.66 \mathrm{a}$ & $34.66 \mathrm{~b}$ & $13.33 \mathrm{c}$ & $38.66 \mathrm{~b}$ & $5.33 \mathrm{c}$ & 35.304 & 0.000 \\
\hline 4-Aspergillus niger & $142.66 \mathrm{ab}$ & $63.33 c$ & 243.00 a & 182.00 bc & $15.00 \mathrm{bc}$ & 21.322 & 0.000 \\
\hline 5-Aspergillus flavus & $18.00 \mathrm{ab}$ & $3.66 \mathrm{c}$ & 25.66 a & $12.00 \mathrm{bc}$ & 9.00 bc & 6.544 & 0.001 \\
\hline 6-Aspergillus terreus & $2.66 \mathrm{~b}$ & 3.00b & $34.00 \mathrm{a}$ & $1.00 \mathrm{~b}$ & $0.330 \mathrm{~b}$ & 11.026 & 0.000 \\
\hline 7-Aspergillus flavipes & $0.00 \mathrm{~b}$ & 0.067b & 38.33a & $2.66 \mathrm{~b}$ & $0.660 \mathrm{~b}$ & $\mathbf{1 7 . 3 1 6}$ & 0.000 \\
\hline 8-Alternaria alternate & $7.33 \mathrm{c}$ & $28.66 \mathrm{~b}$ & 56.00 a & 15.66 bc & $42.66 \mathrm{a}$ & 21.684 & 0.000 \\
\hline 9-Acremonium strictum & $72.66 \mathrm{c}$ & $52.66 \mathrm{c}$ & 144.00a & $114.00 \mathrm{~b}$ & $101.66 \mathrm{~b}$ & 22.533 & 0.000 \\
\hline 10-Rhizopus orrhizus & 6.00b & 3.66b & $7.33 \mathrm{~b}$ & $4.33 \mathrm{~b}$ & 23.33 a & 24.262 & 0.000 \\
\hline 11-Fusarium solani & $4.00 \mathrm{bc}$ & 10.33bc & 55.00 a & $14.66 \mathrm{~b}$ & $0.000 \mathrm{c}$ & 30.411 & 0.000 \\
\hline 12-Absidia corymbifeza & 0.000 b & $0.30 \mathrm{~b}$ & $0.000 b$ & $9.66 \mathrm{a}$ & 0.000 b & 59.107 & 0.000 \\
\hline
\end{tabular}

Where P-value is the probability (reflect of null hypothesis) 
Table ( 3): Frequency and percentage of the isolated yeasts from the collected thirty samples of each of the selected five spices and medicinal plants.

\begin{tabular}{|c|c|c|c|c|c|c|c|c|c|c|c|c|}
\hline \multirow[b]{2}{*}{$\begin{array}{l}\text { Yeast } \\
\text { isolate }\end{array}$} & \multicolumn{2}{|c|}{$\begin{array}{c}\text { Basil } \\
\text { (30 samples ) }\end{array}$} & \multicolumn{2}{|c|}{$\begin{array}{l}\text { Chamomile } \\
\text { (30 samples ) }\end{array}$} & \multicolumn{2}{|c|}{$\begin{array}{c}\text { Dry mint } \\
\text { (30 samples ) }\end{array}$} & \multicolumn{2}{|c|}{$\begin{array}{c}\text { Marjoram } \\
\text { (30 samples ) }\end{array}$} & \multicolumn{2}{|c|}{$\begin{array}{c}\text { Fennel } \\
\text { (30 samples ) }\end{array}$} & \multicolumn{2}{|c|}{$\begin{array}{c}\text { Total } \\
\text { (150 samples) }\end{array}$} \\
\hline & $\begin{array}{c}\text { No. of } \\
\text { isolates }\end{array}$ & $\%$ & $\begin{array}{c}\text { No. of } \\
\text { isolates }\end{array}$ & $\%$ & $\begin{array}{l}\text { No. of } \\
\text { isolates }\end{array}$ & $\%$ & $\begin{array}{c}\text { No. of } \\
\text { isolates }\end{array}$ & $\%$ & $\begin{array}{l}\text { No. of } \\
\text { isolates }\end{array}$ & $\%$ & $\begin{array}{l}\text { No. of } \\
\text { isolates }\end{array}$ & $\%$ \\
\hline Saccharomyces rosinii & 0 & 0.0 & 0 & 0.0 & 0 & 0.0 & 1 & 3.3 & 1 & 3.3 & 2 & 1.3 \\
\hline Candida rugosa & 0 & 0.0 & 2 & 6.6 & 1 & 3.3 & 0 & 0.0 & 0 & 0.0 & 3 & 2.0 \\
\hline Debaromyces hansenii & 0 & 0.0 & 2 & 6.6 & 0 & 0.0 & 0 & 0.0 & 1 & 3.3 & 3 & 2.0 \\
\hline Lipomyces libofore & 0 & 0.0 & 1 & 3.3 & 0 & 0.0 & 1 & 3.3 & 0 & 0.0 & 2 & 1.3 \\
\hline Trichosporon jirovecii & 0 & 0.0 & 1 & 3.3 & 0 & 0.0 & 0 & 0.0 & 0 & 0.0 & 1 & 0.7 \\
\hline Saccharomyces cerevisiae & 0 & 0.0 & 1 & 3.3 & 0 & 0.0 & 0 & 0.0 & 3 & 10.0 & 4 & 2.7 \\
\hline Candida glabrata & 0 & 0.0 & 0 & 0.0 & 0 & 0.0 & 3 & 10.0 & 0 & 0.0 & 3 & 2.0 \\
\hline Total & 0 & $\begin{array}{l}0.0 \\
0.0\end{array}$ & 7 & $\begin{array}{l}23.3 \\
38.9\end{array}$ & 1 & $\begin{array}{l}3.3 \\
5.6\end{array}$ & 5 & $\begin{array}{l}16.7 \\
27.8\end{array}$ & 5 & $\begin{array}{l}16.7 \\
27.8\end{array}$ & 18 & 12 \\
\hline
\end{tabular}


Table (4): Estimation of antifungal activity of ethanolic extracts of three medicinal plants (curcuma, cinnamon and ginger)against the isolated molds from the selected five spices and medicinal plants (basil, chamomile, dry mint, marjoram and fennel).

\begin{tabular}{|c|c|c|c|c|c|c|}
\hline \multirow{2}{*}{ No. } & \multirow{2}{*}{$\begin{array}{c}\text { Sample } \\
\text { code }\end{array}$} & \multirow{2}{*}{ Fungal isolate } & \multirow{2}{*}{$\begin{array}{c}\text { Type of } \\
\text { commodity }\end{array}$} & \multicolumn{3}{|c|}{ Inhibition zone (mm) } \\
\hline & & & & Curcuma & Cinnamon & Ginger \\
\hline 1 & $1.1 \mathrm{~A}$ & Aspergillus glaucus & Basil & 10 & 6 & 2 \\
\hline 2 & $4.20 \mathrm{E}$ & Aspergillus flavus & Marjoram & 0 & 1 & 0 \\
\hline 3 & $1.18 \mathrm{~A}$ & Aspergillus nidulaus & Basil & 0 & 12 & 0 \\
\hline 4 & $1.3 \mathrm{~A}$ & Aspergillus nidulaus & Basil & 0 & 6 & 1 \\
\hline 5 & $1.7 \mathrm{E}$ & Aspergillus flavus & Basil & 0 & 3 & 1 \\
\hline 6 & $2.5 \mathrm{~A}$ & Aspergillus glaucus & Dry mint & 0 & 9 & 1 \\
\hline 7 & $1.5 \mathrm{~A}$ & $\begin{array}{c}\text { Aspergillus } \\
\text { versicolor }\end{array}$ & Basil & 0 & 12 & 0 \\
\hline 8 & $2.23 \mathrm{~A}$ & Alternaria alternata & Dry mint & 3 & 8 & 1 \\
\hline 9 & $2.50 \mathrm{~A}$ & Alternaria alternata & Dry mint & 2 & 17 & 1 \\
\hline 10 & $1.24 \mathrm{E}$ & Aspergillus flavus & Basil & 0 & 4 & 1 \\
\hline 11 & 4.8D & Aspergillus niger & Marjoram & 2 & 0 & 1 \\
\hline 12 & $1.10 \mathrm{~A}$ & Aspergillus nidulaus & Basil & 2 & 5 & 1 \\
\hline 13 & $1.3 \mathrm{~A}$ & $\begin{array}{c}\text { Aspergillus } \\
\text { versicolor }\end{array}$ & Basil & 0 & 6 & 0 \\
\hline 14 & $4.10 \mathrm{~A}$ & $\begin{array}{c}\text { Aspergillus } \\
\text { versicolor }\end{array}$ & Marjoram & 2 & 17 & 1 \\
\hline 15 & $2.33 \mathrm{~A}$ & Aspergillus glaucus & Dry mint & 0 & 22 & 0 \\
\hline 16 & $2.37 \mathrm{~A}$ & Alternaria alternata & Dry mint & 0 & 10 & 1 \\
\hline 17 & $2.36 \mathrm{~A}$ & $\begin{array}{c}\text { Aspergillus } \\
\text { versicolor }\end{array}$ & Dry mint & 2 & 15 & 1 \\
\hline 18 & $2.38 \mathrm{~A}$ & Alternaria alternata & Dry mint & 0 & 19 & 0 \\
\hline 19 & $1.16 \mathrm{~A}$ & Alternaria alternata & Basil & 0 & 10 & 3 \\
\hline 20 & 2.14B & Acremonium strictum & Dry mint & 5 & 4 & 0 \\
\hline 21 & $6.2 \mathrm{~B}$ & Acremoniumstrictum & Fennel & 0 & 2 & 0 \\
\hline 22 & $2.18 \mathrm{G}$ & Fusarium solani & Dry mint & 0 & 3 & 0 \\
\hline 23 & $2.8 \mathrm{~B}$ & Acremoniumstrictum & Dry mint & 2 & 8 & 0 \\
\hline 24 & $5.8 \mathrm{G}$ & Fusarium solani & Chamomile & 0 & 10 & 1 \\
\hline 25 & $2.16 \mathrm{G}$ & Fusarium solani & Dry mint & 0 & 9 & 1 \\
\hline 26 & $4.4 \mathrm{~F}$ & Rhizopus orrhizus & Marjoram & 4 & 2 & 3 \\
\hline 27 & $1.11 \mathrm{~B}$ & $\begin{array}{c}\text { Acremonium } \\
\text { strictum }\end{array}$ & Basil & 0 & 6 & 2 \\
\hline 28 & $5.9 \mathrm{~F}$ & Rhizopus orrhizus & Chamomile & 2 & 0 & 0 \\
\hline 29 & $2.2 \mathrm{G}$ & Fusarium solani & Dry mint & 0 & 6 & 0 \\
\hline 30 & $2.5 \mathrm{G}$ & Fusarium solani & Dry mint & 0 & 12 & 0 \\
\hline 31 & $2.42 \mathrm{~A}$ & Alternaria alternata & Dry mint & 0 & 14 & 2 \\
\hline 32 & $5.29 \mathrm{~A}$ & Alternaria alternata & Chamomile & 0 & 2 & 2 \\
\hline 33 & $2.15 \mathrm{~F}$ & Rhizopus orrhizus & Dry mint & 2 & 0 & 2 \\
\hline 34 & $1.4 \mathrm{~B}$ & Acremonium strictum & Basil & 0 & 5 & 0 \\
\hline 35 & $2.46 \mathrm{~A}$ & Alternaria alternata & Dry mint & 2 & 9 & 2 \\
\hline 36 & $2.36 \mathrm{~A}$ & Alternaria alternata & Dry mint & 3 & 11 & 1 \\
\hline
\end{tabular}


Cont. Table (4):

\begin{tabular}{|c|c|c|c|c|c|c|}
\hline \multirow[t]{2}{*}{ No. } & \multirow{2}{*}{$\begin{array}{l}\text { Sample } \\
\text { code }\end{array}$} & \multirow[t]{2}{*}{ Fungal isolate } & \multirow{2}{*}{$\begin{array}{c}\text { Type of } \\
\text { commodity }\end{array}$} & \multicolumn{3}{|c|}{ Inhibition zone (mm) } \\
\hline & & & & Curcuma & Cinnamon & Ginger \\
\hline 37 & $5.16 \mathrm{~A}$ & $\begin{array}{c}\text { Alternaria } \\
\text { alternata }\end{array}$ & Chamomile & 0 & 15 & 2 \\
\hline 38 & $2.9 \mathrm{~B}$ & $\begin{array}{l}\text { Acremonium } \\
\text { strictum }\end{array}$ & Dry mint & 0 & 6 & 3 \\
\hline 39 & 2.44 & $\begin{array}{c}\text { Absidia } \\
\text { corymbifeza }\end{array}$ & Dry mint & 2 & 16 & 1 \\
\hline 40 & $5.27 \mathrm{E}$ & Aspergillus flavus & Chamomile & 0 & 13 & 1 \\
\hline 41 & $6.26 \mathrm{~B}$ & $\begin{array}{c}\text { Acremonium } \\
\text { strictum }\end{array}$ & Fennel & 0 & 5 & 4 \\
\hline 42 & $1.2 \mathrm{G}$ & Fusarium solani & Basil & 0 & 3 & 0 \\
\hline 43 & $1.2 \mathrm{~B}$ & $\begin{array}{l}\text { Acremonium } \\
\text { strictum }\end{array}$ & Basil & 0 & 16 & 4 \\
\hline 44 & 2.17D & Aspergillus niger & Dry mint & 0 & 0 & 1 \\
\hline 45 & $2.50 \mathrm{D}$ & Aspergillus niger & Dry mint & 0 & 0 & 0 \\
\hline 46 & $1.2 \mathrm{~F}$ & Rhizopus orrhizus & Basil & 0 & 1 & 2 \\
\hline 47 & $6.17 \mathrm{~A}$ & $\begin{array}{l}\text { Alternaria } \\
\text { alternata }\end{array}$ & Fennel & 0 & 13 & 3 \\
\hline 48 & $6.18 \mathrm{~B}$ & $\begin{array}{l}\text { Acremonium } \\
\text { strictum }\end{array}$ & Fennel & 0 & 12 & 0 \\
\hline 49 & $2.25 \mathrm{I}$ & $\begin{array}{l}\text { Aspergillus } \\
\text { flavipes }\end{array}$ & Dry mint & 0 & 2 & 0 \\
\hline 50 & $4.25 \mathrm{~B}$ & $\begin{array}{c}\text { Acremonium } \\
\text { strictum }\end{array}$ & Marjoram & 0 & 11 & 0 \\
\hline 51 & $2.33 \mathrm{E}$ & Aspergillus terreus & Dry mint & 0 & 9 & 0 \\
\hline 52 & $4.19 \mathrm{~A}$ & $\begin{array}{l}\text { Aspergillus } \\
\text { glaucus }\end{array}$ & Marjoram & 2 & 0 & 2 \\
\hline 53 & 4.27 & $\begin{array}{c}\text { Absidia } \\
\text { corymbifeza }\end{array}$ & Marjoram & 0 & 7 & 6 \\
\hline 54 & $2.4 \mathrm{~F}$ & Rhizopus orrhizus & Dry mint & 5 & 2 & 2 \\
\hline 55 & $1.14 \mathrm{~F}$ & Rhizopus orrhizus & Basil & 0 & 0 & 2 \\
\hline 56 & $5.27 \mathrm{~B}$ & $\begin{array}{l}\text { Acremonium } \\
\text { strictum }\end{array}$ & Chamomile & 0 & 13 & 1 \\
\hline 57 & $2.26 \mathrm{~B}$ & $\begin{array}{c}\text { Acremonium } \\
\text { strictum }\end{array}$ & Dry mint & 0 & 15 & 0 \\
\hline 58 & $2.42 \mathrm{~F}$ & Rhizopus orrhizus & Dry mint & 0 & 2 & 1 \\
\hline 59 & $2.7 \mathrm{D}$ & Aspergillus niger & Dry mint & 0 & 0 & 0 \\
\hline 60 & $2.47 \mathrm{I}$ & $\begin{array}{l}\text { Aspergillus } \\
\text { flavipes }\end{array}$ & Dry mint & 0 & 22 & 3 \\
\hline
\end{tabular}

Ginger contains a vast and complex array of chemicals that, in combination, provides a powerful aid to healing. The enzyme zingibain was believed to improve digestion as well as kills parasites and their eggs. Furthermore, zingibain enhanced antibacterial and antifungal actions ( Tajkarimi et al., 2010).

There was a significant difference in using the ethanolic extract of each of Curcuma, Cinnamon and Ginger against Aspergillus versicolor, A.nidulans, Acremonium strictum and Fusarium solani ( the mean values of inhibition zones take different letters) (Table,5).

3.3. Estimation of mycotoxins production from fungal contaminants of the selected five spices and medicinal plants

Mycotoxin contamination is a worldwide food safety problem. It can cause significant economic 
Table (5): Estimation of antifungal activity of ethanolic extracts of three medicinal plants (curcuma, cinnamon and ginger)against the isolated yeasts from the selected five spices and medicinal plants (basil, chamomile, dry mint, marjoram and fennel).

\begin{tabular}{|c|c|c|c|c|c|c|}
\hline \multirow[t]{2}{*}{ No. } & \multirow{2}{*}{$\begin{array}{c}\text { Code } \\
\text { number }\end{array}$} & \multirow[t]{2}{*}{ Yeast isolate } & \multirow{2}{*}{$\begin{array}{c}\text { Type of } \\
\text { commodity }\end{array}$} & \multicolumn{3}{|c|}{$\begin{array}{c}\text { Inhibition zone (mm)using ethanolic extract } \\
\text { of }\end{array}$} \\
\hline & & & & Curcuma & Cinnamon & Ginger \\
\hline 1 & 4.6 & Lipomyces lipofore & Marjoram & 0 & 2 & 10 \\
\hline 2 & 2.11 & Candida rugosa & Dry mint & 0 & 1 & 12 \\
\hline 3 & 5.23 & Candida rugosa & Chamomile & 0 & 6 & 17 \\
\hline 4 & 5.9 & Lipomyces lipofore & Chamomile & 0 & 2 & 15 \\
\hline 5 & 5.10 & Saccharomyces cerevisiae & Chamomile & 0 & 0 & 19 \\
\hline 6 & 5.3 & Candida rugosa & Chamomile & 0 & 0 & 23 \\
\hline 7 & 5.9 & Trichosporon Jirovecii & Chamomile & 0 & 3 & 20 \\
\hline 8 & 5.30 & Debaromyces hansenii & Chamomile & 0 & 1 & 30 \\
\hline 9 & 5.8 & Debaromyces hansenii & Chamomile & 0 & 1 & 10 \\
\hline 10 & 6.28 & Saccharomyces cerevisiae & Fennel & 2 & 3 & 15 \\
\hline 11 & 6.15 & Debaromyces hansenii & Fennel & 3 & 3 & 16 \\
\hline 12 & 6.26 & Saccharomyces cerevisiae & Fennel & 4 & 3 & 15 \\
\hline 13 & 6.16 & Saccharomyces cerevisiae & Fennel & 0 & 5 & 26 \\
\hline 14 & 6.17 & Saccharomyces rosinii & Fennel & 0 & 3 & 20 \\
\hline 15 & 4.10 & Candida glabrata & Marjoram & 0 & 0 & 15 \\
\hline 16 & 4.6 & Candida glabrata & Marjoram & 0 & 1 & 15 \\
\hline 17 & 4.1 & Candida glabrata & Marjoram & 2 & 0 & 15 \\
\hline 18 & 4.5 & Saccharomyces rosinii & Marjoram & 0 & 0 & 25 \\
\hline
\end{tabular}

Table ( 6): Mean value of antifungal activity(inhibition zone) of three medicinal plants ethanolic extracts (curcuma, cinnamon and ginger)against the isolated molds from the selected five spices and medicinal plants (basil, chamomile, dry mint, marjoram and fennel).

\begin{tabular}{|c|c|c|c|c|c|}
\hline \multirow[b]{2}{*}{ Fungal isolate } & \multicolumn{3}{|c|}{ Inhibition zone (mm) } & \multicolumn{2}{|c|}{ F-test } \\
\hline & Curcuma & Cinnamon & Ginger & F-value & $\mathrm{P}$-value \\
\hline 1-Aspergillus glaucus & $000.3 \mathrm{a}$ & $9.250 \mathrm{a}$ & $1.250 \mathrm{a}$ & 1.932 & 0.200 \\
\hline 2-Aspergillus versicolor & $1.000 \mathrm{~b}$ & $12.500 \mathrm{a}$ & $0.500 \mathrm{~b}$ & 22.419 & 0.000 \\
\hline 3-Aspergillus nidulaus & $1.000 \mathrm{~b}$ & $5.500 \mathrm{a}$ & $1.000 \mathrm{~b}$ & 16.200 & 0.020 \\
\hline 4-Aspergillus niger & $0.500 \mathrm{a}$ & $0.000 \mathrm{a}$ & $0.250 \mathrm{a}$ & 0.600 & 0.560 \\
\hline 5-Aspergillus flavus & $0.000 \mathrm{a}$ & $5.250 \mathrm{a}$ & $0.750 \mathrm{a}$ & 3.395 & 0.080 \\
\hline 7-Aspergillus flavipes & $0.000 \mathrm{a}$ & $12.000 \mathrm{a}$ & $1.500 \mathrm{a}$ & 1.254 & 0.400 \\
\hline 8-Alternaria alternate & $0.9090 \mathrm{a}$ & $11.6364 \mathrm{a}$ & $1.6364 \mathrm{~b}$ & 48.204 & 0.000 \\
\hline 9-Acremonium strictum & $0.5830 \mathrm{~b}$ & $8.5833 \mathrm{a}$ & $1.667 \mathrm{~b}$ & 27.022 & 0.000 \\
\hline 10-Rhizopus orrhizus & $1.8571 \mathrm{a}$ & $1.0000 \mathrm{a}$ & $1.7143 \mathrm{a}$ & 0.7320 & 0.490 \\
\hline 11-Fusarium solani & $0.000 \mathrm{~b}$ & $7.167 \mathrm{a}$ & $0.330 \mathrm{~b}$ & 20.404 & 0.000 \\
\hline 12-Absidia corymbifeza & $1.000 \mathrm{a}$ & $11.5000 \mathrm{a}$ & $3.500 \mathrm{a}$ & 3.282 & 0.170 \\
\hline
\end{tabular}


losses associated with the adverse effects of mycotoxins on human and animal health, food security and international trade. Mycotoxins are toxic substances produced mostly as secondary metabolites by fungi that grow on seeds and feed in the field, or in storage (Trucksess and DiazAmigo, 2011). The major mycotoxin-producing fungi are species of Aspergillus, Fusarium and Penicillium (Kumar et al., 2008). In the present study, seven mold isolates out of sixty produced mycotoxins (11.7\%). Aspergillus flavus from Marjoram produced aflatoxin $\mathrm{B}_{1}(160 \mu \mathrm{g} / \mathrm{ml})$ and aflatoxin $\mathrm{B}_{2}(50 \mu \mathrm{g} / \mathrm{ml})$, while,each of A.glaucus, A.flavus from Basil and A.niger from Marjoram produced aflatoxin $\mathrm{G}_{1}$ as $3.3 \mu \mathrm{g} / \mathrm{ml}, 25 \mu \mathrm{g} / \mathrm{ml}$ and $7.5 \mu \mathrm{g} / \mathrm{ml}$,respectively(Fig,1). Each of A.glaucus, A.versicolor from Dry mint and A.nidulaus from basil produced Zeralenone. A significant part of aflatoxin B1 in the Egyptian food could come from nine essential food spices and dried herbs, most of them are probably infested with Aspergillus flavus during drying and storage (Selim et al.,1996 \& Kumar et al., 2009). There had been a growing body of circumstantial evidence that aflatoxin $B_{1}$ is carcinogenic, as well as acutely toxic to humans, but in 1987 the International Agency for Research on Cancer (IARC) and the World Health Organization (WHO) accepted that aflatoxin should be classified as a Group 1 carcinogen. Regulatory levels set by the governments of many countries were based on the premise that aflatoxin is indeed carcinogenic and the European Community agreed on 16 July 1998 a limit of $2 \mu \mathrm{g} \mathrm{kg}^{-1}$ for aflatoxin $\mathrm{B}_{1}$ in a range of foods for human consumption. Bungo et al. (2006)and Ajose (2007) reported that, a potential risk for mycotoxins contamination may occur especially during prolonged storage in poor condition without tempreture and moisture control that usually render medicinal plants more susceptible to mould growth and mycotoxins production.

\section{REFERENCES}

Abou Donia M.A. (2008). Microbiological Quality and Aflatoxinogenesis of Egyptian Spieces and Medicinal plants. Depatrment of Food Toxicology and Contaminants, National Research Center.2(4): 175-181.

Ahearn D.G. (1978). Medically important yeasts. Ann. Rev. Micrbiol. 32: 59-68.

Ajose (2007). Some Nigerian plants of dermatologic importance. Int. J. Dermatol., 46 (s1): 48-55.

Armitage P. (1971). Statistical Methods in
Medical Research. Blackwell Scientific Publications London.

Aziz N. H., Youssef Y. A., El-Fouly M. Z. and Moussa L.A. (1998). Contamination of some common medicinal plant samples and spices by fungi and their mycotoxins. Bot. Bull. Acad. Sinica .39: 279-285.

Barnett J.A., Payne R.W. and Yarrow D. (2000). Yeasts: characteristics and identification, Third edition, Cambridge University Press, Cambridge, UK 1139, pp 5320.

Bauer A.W., Kirby W.M., Sherris J.C. and Turck, M. (1966). Antibiotic susceptibility testing by a standardized single disk method. Am. J. Clin. Pathol.45,493-496.

Bungo A., Almodovar A. B., Pereira T.C., PintoT.A. and Sabino M. (2006). Occurrence of toxigenic fungi in herbal drugs. Brazilian Journal of Microbiology. 37(1):47-51.

Clark A.M., El-Feraly A.S. and Wen-Shyong Li. (1981). Antimicrobial activity of phenolic constituents of Magnolia grandiflora L. Journal of Pharmaceutical Sciences.70(8):951952.

Dubey N. K., Kumar, A., Singh P. and Shukla R. (2008). Microbial contamination of raw materials: A major reason for the decline of India's share in the global herbal market. 95(6):717-718.

El-Sawi A. M. (2000). Studies on Contaminants, Monitoring and Fate of Pesticides in some Medicinal plants. Ph.D Thesis, Faculty of Agriculture, Cairo University, Egypt.

El Tahan F.H. and Fahmy S.M. (2005). Monitoring of microbial and pesticide residues contamination in the unpacked and packed medicinal plants distributed for local consumption. Journal of the Advances Agricultureal Researches. 10(3): 675-688.

El Tahan F.H. and Taha M. (1996). Study on toxicity and carcinogenicity of aflatoxin in laboratory animals. Ph.D. Thesis.Faculty of Veterinary Medicine, Zagazig University, Egypt.

Hashem M. and Alamri S. (2010). Contamination of common spices in Saudi Arabia markets with potential mycotoxin-producing fungi. Saudi Journal of Biological Sciences. 17 (2): $167-175$.

ISO 21527 (2001). Microbiology of food and animal feeding stuffs-Horisontal method for the enumeration of yeasts and moulds.

John I., (1979). The genus Penicillium and its telemorphic states Eupenicillium and Talaromyces. London, Academic Press. [ISBN 
0125577507].

Kendrick B. (2000). The Fifth Kingdom, the third edition, Focus Publishing, R. Paulins Company, Newburyport Massachusetts, USA. [ISBN 1-58510-022-6].

Kneifel W. and Berger E. (1994). Microbial criteria of random samples of spices and herbs retailed on the Austrian market. J. Food Protect. 57: 893-901.

Kneifel W., Czech E. and Kopp B. (2002). Microbial contamination of medical plants. Planta- Medica.;68(1): 5-15.

Kumar V., Basu M.S. and Rajendran T.P. (2008). Mycotoxin research and mycoflora in some commercially important agricultural commodities.Crop Protection. 27 (6): 891-905.

Kumar A., Shukla R., Singh P. and Dubey N.K. (2009). Biodeterioration of some herbal raw materials by storage fungi and aflatoxin and assessment of Cymbopogonflexuosus essential oil and its components as antifungal. International Biodeterioration \& Biodegradation. 63(6): 712-716.

Lodder J. (1971). The Yeasts. A taxonomic study. North-Holland Publ. Co., Amsterdam.

Lopez P., Sanchez C., Batle R. and Nerin C. (2005). Solid- and vapor-phaseantimicrobial activities of six essential oils: susceptibility of selected foodborne bacterial and fungal strains.Journal of Agricultural and Food Chemistry. 53(17): 6939-6946.

Mandeel Q.A. (2005). Fungal contamination of some imported spices. Mycopathologia, 159: 291-298.

Martins H.M.,Martins M.L., Dias M.I. and Bernardo F. (2001). Evaluation of microbiological quality of medicinal plants used in natural infusions. International Journal of Food Microbiology. 68(1-2), 15:149-153.

McKee L.H. (1995). Microbial contamination of spices and herbs: A review LWT - Food Science and Technology. 28 (1): 1-11.

Samson R.A. (1974). Paecilomyces and some allied hypomycetes. Stud. Mycol. 6:1-119.
Selim M.I., Pependorf F.W., Ibrahim M.S., ElSarkawy S. and El-kashory E.S. (1996). Aflatoxin B1 in common Egyptian foods. Journal of AOAC. 79,1124-1129.

Simić A., Soković M., Ristić M., GrujićJovanović S., Vukojević J. and Marin P. (2004). The chemical composition of some Lauracae essential oils and their antifungal activities Phytotherapy Research. 18(9): 713717.

Singh G., Singh P. and Maurya S. (2002). Chemical and biocidalinvestigations on essential oils of some Indian Curcuma species. Progress in Crystal Growth and Characterization of Materials.45(1-2):75-81.

Tajkarimi M.M., Ibrahim S.A. and Cliver D.O. (2010). Antimicrobial herb and spice compounds in food. Food Control., 21 (9): 1199-1218.

Trucksess M.W. and Diaz-Amigo C. (2011). Mycotoxins in Foods. Encyclopedia of Environmental Health. Pages 888-897.

Velickvic D., Randjelovic N., Ristic M., Smelceovic A. and Velickvic A. (2002). Chemical composition of the ethanolic extracts of Salivia pratensis L., S. glutinosa L. and S. aethiopis L. J. Serb. Chem. Soc., 67:639-646.

Walker R. (2002). Risk assessment of ochratoxin: current views of the European scientific committee on food, the JECFA and the codex committee on food additives and contaminants Adv. Exp. Med. Biol. 504:24955.

Wickerham L.J. (1951). Taxonomy of yeasts., US. Dept. Agriculture Technical Bulletin No. 1029: 1-56.

Wojcik S. B. and Jakubowska B. (2005). Estimation of microbiological quality of spices delivered by various producers. Folia Universitatis Agreculturae Stetinensis, Scientia-Alimentaria. 246(4):303-311. 
المحتوى الفطرى لبعض النباتات الطبية والبهارات المصرية وتقييم قدراتها لانتاج السموم الفطرية

فؤاد حلمى الطحان - يسرية محمد حسن شتيه * ـ منال فوزى عبد العزيز

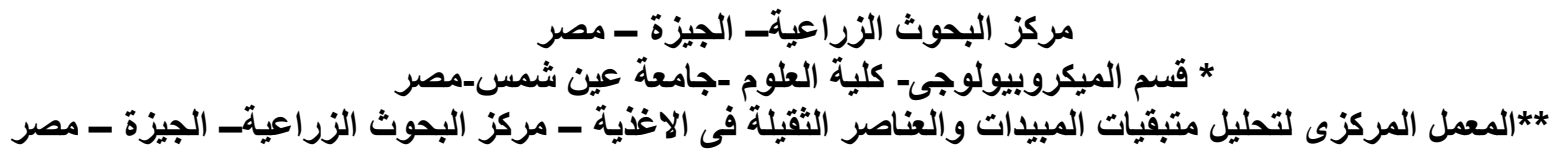

ملخص بهرار

تم عمل مسح على مائة و خمسون عينة من الخمس بهار ات و النباتات الطبية ( ثلاثون عينة من كل من الريحان

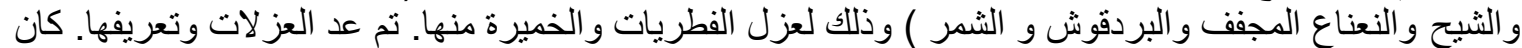

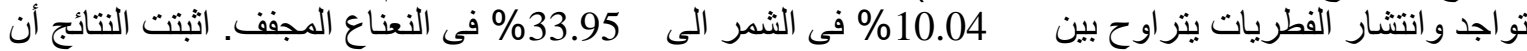

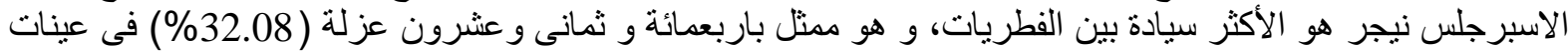

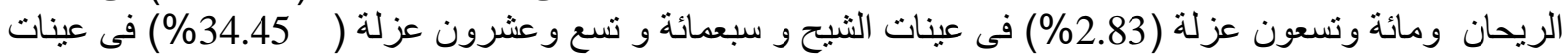

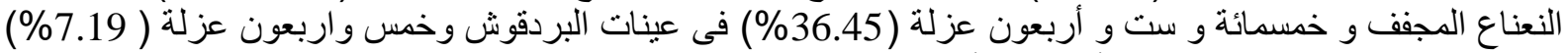

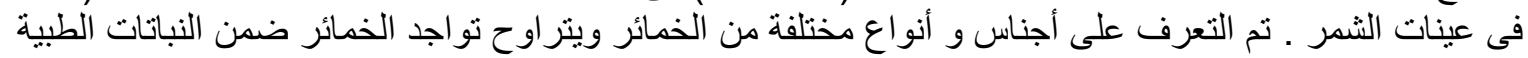

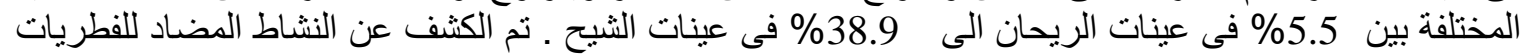

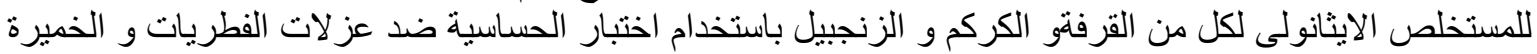

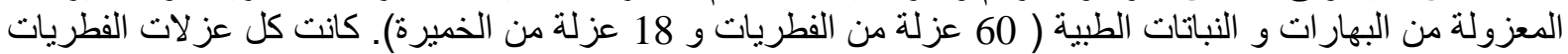

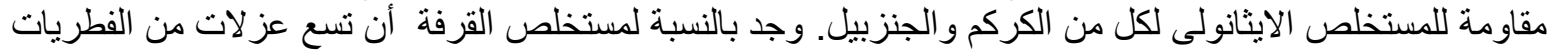

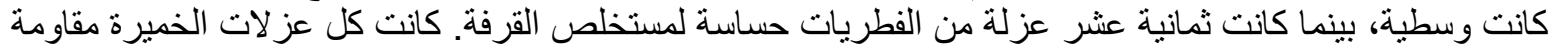

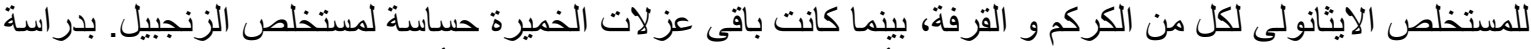

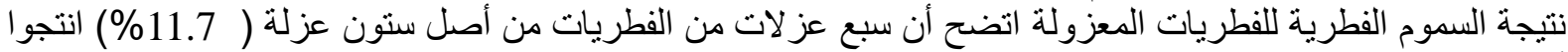

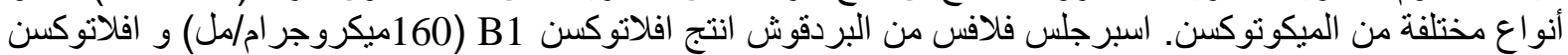
50)B2

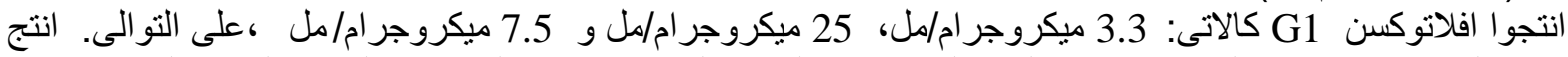

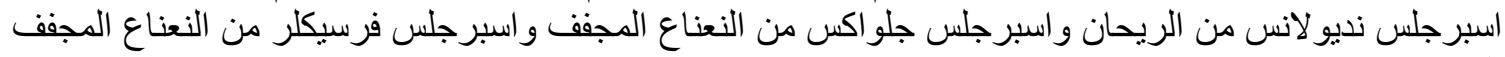

المجلة العلمية لكلية الزراعة - جامعة القاهرة - المجلد (63) العدد الثالث (يولية2012):344-333. 